O. Neufang (Hrsg.)

Lexikon der Elektronik 


\section{LEXIKON DER ELEKTRONIK}

Herausgeber: Prof. Dr. rer. nat. O. Neufang

Unter Mitarbeit von:

Prof. Dr. rer. nat. B. Blum, Rhein. Fachhochschule Köln

Dipl.-Ing. W. Fleischhauer, Rhein. Fachhochschule Köln

Prof. Dipl.-Ing. U. Gerlach, Fachhochschule Düsseldorf

Prof. Dr.-Ing. P. Kühn, Universität Stuttgart

Prof. Dr.-Ing. U. Kunz, Universität-GH-Siegen

Dipl.-Phys. W. Link, b.i.b. Paderborn

Prof. Dr. rer. nat. H. Rühl, Universität-GH-Siegen

Dipl.-Ing. A. Thiel, PTB Braunschweig

Dipl.-Ing. P. Welzel, b.i.b. Paderborn 
O. NEUFANG (Hrsg.)

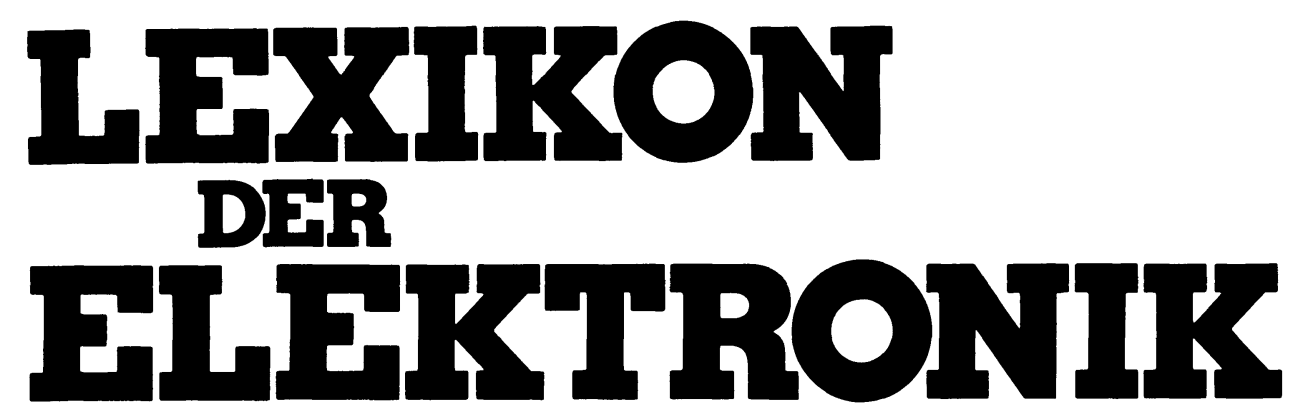

Mit 676 Abbildungen

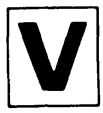

Friedr. Vieweg \& Sohn Braunschweig / Wiesbaden 
Alle Rechte vorbehalten

() Friedr. Vieweg \& Sohn Verlagsgesellschaft mbH, Braunschweig 1983

Softcover reprint of the hardcover 1st edition 1983

Die Vervielfältigung und Übertragung einzelner Textabschnitte, Zeichnungen oder Bilder, auch für Zwecke der Unterrichtsgestaltung, gestattet das Urheberrecht nur, wenn sie mit dem Verlag vorher vereinbart wurden. Im Einzelfall muß über die Zahlung einer Gebühr für die Nutzung fremden geistigen Eigentums entschieden werden. Das gilt für die Vervielfältigung durch alle Verfahren einschließlich Speicherung und jede Übertragung auf Papier, Transparente, Filme, Bänder, Platten und andere Medien.

Satz: Vieweg, Braunschweig Druck: CW Niemeyer, Hameln

Buchbinderische Verarbeitung: Hunke \& Schröder, Iserlohn 


\section{Vorwort}

Mit Erfindung des Bipolartransistors am 24.12.1947 wurde eine neue Epoche der Elektronik eingeleitet, die zur Entwicklung von Halbleiterdioden, verbesserten Strukturen von Bipolartransistoren, Bauelementen mit Metall-Halbleiterübergängen, Feldeffekttransistoren, optoelektronischen Bauelementen, integrierten Strukturen u.a. m. führte. Heute ist man in der Lage, auf einem Halbleiterplättchen von wenigen Millimetern Kantenlänge mehr als 200000 Einzelbauelemente unterzubringen. Die Entwicklungen der Zukunft streben noch höhere Integrationsdichten an. Insbesondere wird man jedoch bemüht sein, die Verzögerungszeiten einer Einzelstruktur in einer integrierten Schaltung zu verringern. Nachdem die minimale Verzögerungszeit seit nun über 10 Jahren bei etwa 1 ns liegt, wird es vielleicht schon Mitte der 80er Jahre kommerzielle Bausteine mit Verzögerungszeiten im Bereich 50 ps geben.

Ein Lexikon der Elektronik darf sich jedoch nicht nur der Halbleiterelektronik und ihren Bauelementen widmen. Es müssen auch deren Grundlagen behandelt werden. Hierzu gehört die Darstellung sowohl der elektrotechnischen Grundlagen (im weitesten Sinne) als auch der Halbleiterphysik.

Wenn man bedenkt, daß in den 70er Jahren mehr als 40000 Zeitschriftenartikel auf dem Halbleitergebiet veröffentlicht wurden (im Jahre 1970 waren es 2700 und im Jahre 1979 5500 mit steigender Tendenz), kann ein einbändiges Lexikon der Elektronik nur die wichtigsten Begriffe, die zum verständnisvollen Lesen der Fachliteratur notwendig sind, abhandeln.

Das vorliegende Lexikon der Elektronik gliedert sich in folgende vier Abschnitte:

- Einen lexikographischen Teil, in dem die Fachgebiete Angewandte Elektronik, Digitalelektronik, Elektrische Energietechnik, Elektrische Filter, Elektrische Meßtechnik, Elektronische Bauelemente, Elektronische Datenverarbeitung, Elektrophysik, Feldtheorie, Halbleiterelektronik, Halbleiterphysik, Höchstfrequenztechnik, Informationsverarbeitung, Integrierte Schaltungen, Nachrichtentechnik, Nachrichtenübertragungstechnik, Nachrichtenvermittlung, Netzwerktheorie, Optoelektronik, Prozeßrechentechnik, Regelungstechnik behandelt werden.

- Ein Literaturverzeichnis, in dem aus Platzgründen ausschließlich Bücher aufgeführt sind.

- Ein englisch-deutsches Begriffslexikon, das für die größte Anzahl der im lexikographischen Teil abgehandelten deutschen Begriffe ein englisches Äquivalent enthält. Das Wörterbuch soll auch dazu dienen, beim Lesen englischsprachiger Literatur die Bedeutung unbekannter Begriffe nachlesen zu können.

- Einen Anhang mit englischsprachigen Abkürzungen, die sonst nur über Spezialliteratur ermittelt werden können. 
Es wurde versucht, die Begriffe und Definitionen mit den Normen des DIN bzw. anderer Fachgesellschaften (z. B. NIG, IEEE) in Einklang zu bringen. Da sich die Terminologie technisch aktueller Gebiete ständig ändert, befinden sich an verschiedenen Stellen beschriebene Normen nicht immer in voller Übereinstimmung. Beim Zitieren von DINNormen wurde die manchmal schwerfällige sprachliche Formulierung übernommen.

Obwohl für viele angloamerikanischen Begriffe ein deutschsprachiges Äquivalent besteht, wurden die angloamerikanischen Begriffe - oft in den gewohnten Abkürzungen - aufgenommen, weil sie (bedauerlicherweise) in der deutschen Fachliteratur Eingang gefunden haben.

Der Herausgeber ist den Mitarbeitern - ein jeder Spezialist auf den von ihm bearbeiteten Gebieten - dieses Elektroniklexikons zu tiefem Dank verpflichtet, da jeder einzelne sehr viele Stunden seiner Freizeit geopfert hat. Dem Vieweg Verlag und insbesondere dem Lektor Herrn A. Schubert sei für den reibungslosen Ablauf bei der Entstehung dieses Lexikons gedankt.

Es ist dem Verlag und dem Herausgeber klar, daß bei der Breite der Gebiete und dem Umfang des Lexikons einzelne Wünsche offenbleiben müssen. Wir sind deshalb sehr dankbar, wenn aus dem Leserkreis Anregungen hinsichtlich Ergänzungen und Verbesserungen des Lexikons der Elektronik als einbändiges Werk an uns herangetragen werden.

O. Neufang

Im März 1983 


\section{Hinweise für den Benutzer}

1. Die Stichworte sind alphabetisch aufgeführt.

2. Umlaute werden wie die Buchstaben a, o, u behandelt. Beispiel: „Flüssigkeit“ steht zwischen ,Flußdichte“ und „Flußmesser“.

3. Zusammengesetzte Stichworte sind unter dem Substantiv zu finden. Beispiel: „differentielle Permeabilität“ wird unter „Permeabilität, differentielle“ abgehandelt.

4. Wissenschaftliche Bezeichnungen, die mit dem Begriff „Photo“ zusammenhängen, sind unter ,Ph“, nicht unter ,,F“ aufgeführt.

5. Pfeile besagen, daß das mit Pfeil versehene Stichwort gesondert abgehandelt wird.

6. Geschützte Warenzeichen der Handelsnamen sind nicht besonders gekennzeichnet.

7. Abkürzungen werden in Anlehnung an den Duden verwendet.

8. Bei den im Literaturverzeichnis aufgeführten Jahreszahlen handelt es sich jeweils um das Erscheinungsjahr der letzten Auflage.

Sie finden:

Lexikographischer Teil

Seite 1

Literaturverzeichnis

Englisch-deutsches Begriffslexikon Seite 707

Englischsprachige Abkürzungen Seite 757 


\section{Verzeichnis der Mitarbeiter, ihre Kürzel sowie die von innen bearbeiteten Sachgebiete}

Prof. Dr. rer. nat. B. Blum (Bl)

Dipl.-Ing. W. Fleischhauer (Fl)

Prof. Dipl.-Ing. U. Gerlach (Ge)

Prof. Dr.-Ing. Kühn (Kü)

Prof. Dr.-Ing. U. Kunz (Ku)

Dipl.-Phys. W. Link (Li)

Prof. Dr. rer. nat. O. Neufang $(\mathrm{Ne})$

Prof. Dr. rer. nat. H. Rühl (Rü)

Dipl.-Ing. A. Thiel (Th)

Dipl.-Ing. P. Welzel (We)
Halbleiterelektronik, Halbleiterphysik

Angewandte Elektronik, Elektrische Meßtechnik

Drahtlose Nachrichtenübertragungstechnik, Höchstfrequenztechnik, Passive Elektronische Bauelemente

Nachrichtenvermittlung, Rechner- und Datenkommunikation

Elektrische Energietechnik, Regelungstechnik

Digitalelektronik, Elektronische Datenverarbeitung, Elektronische Schaltungstechnik, Informationsverarbeitung, Integrierte Schaltungen, Optoelektronik

Aktive Elektronische Bauelemente, Integrierte Schaltungen, Optoelektronik

Elektrische Filter, Elektrophysik, Feldtheorie, Grundlagen der Elektrotechnik, Netzwerktheorie, Nachrichtentechnik (allgemein),

Drahtgebundene Nachrichtentechnik, Nachrichtenübertragungstechnik

Digitaltechnik, Elektronische Datenverarbeitung, Informationsverarbeitung, Prozeßrechentechnik 